\title{
COMPLEJIDAD E IMPEDIMENTOS EN EL NIVEL FÍSICO PARA LA IMPLEMENTACION DE REDES SATELITALES COGNITIVAS
}

\author{
COMPLEXITY AND IMPEDIMENTS IN THE PHYSICAL LEVEL FOR THE \\ IMPLEMENTATION OF COGNITIVE SATELLITE NETWORKS
}

Evelio Astaiza Hoyos $^{1}$, Héctor Fabio Bermúdez Orozco ${ }^{1}$

${ }^{1}$ Programa de Ingeniería Electrónica de la Universidad del Quindío, eastaiza@uniquindio.edu.co, hfbermudez@uniquindio.edu.co

Recibido: Marzo 5 de 2012

Aceptado: Mayo 10 de 2012

*Correspondencia del autor. Grupo de Investigación en Telecomunicaciones de la Universidad del Quindío, gituq@uniquindio.edu.co, tel 7460298 ext 108. Centro de Estudios e Investigaciones de la Facultad de Ingeniería - CEIFI Universidad del Quindío. Carrera 15 Nro.

\section{RESUMEN}

En este artículo se muestran algunos impedimentos que se presentan en la actualidad en el nivel físico para la implementación de redes satelitales cognitivas, adicionalmente se ilustra desde la perspectiva de la teoría de juegos como pueden ser implementadas de manera óptima algunas de las funciones cognitivas requeridas en este tipo de sistemas. Las funciones básicas de un satélite y/o estación terrena cognitiva son flexibilidad, reconfigurabilidad, autoconfigurabilidad, acceso dinámico de espectro, adaptación y aprendizaje de las condiciones del entorno para la optimización en la utilización de los recursos de radio; algunas de estas funciones pueden implementarse a través de software radio, sin embargo la gran mayoría de ellas requieren capacidades de procesamiento elevadas, altos costos computacionales y tiempos de respuesta muy bajos, lo cual en muchos casos impide su implementación.

Palabras Claves: Aprendizaje, Asignación de Recursos, Calidad de Servicio, Radio Cognitivo, Radio Definido por Software, DSP, FPGA, Redes Satelitales Cognitivas, Estación Base.

\begin{abstract}
In this paper some impediments that are currently on the physical level to implement cognitive satellite networks are show. Further illustrated from the standpoint of game theory as can be optimally implemented some of the cognitive functions required in these systems. The basic functions of a satellite and / or cognitive earth station are: flexibility, reconfigurability, auto-configurability, dynamic spectrum access, adaptation and learning of environmental conditions to optimize the use of radio resources. Some of these functions can be implemented through radio software, however, the most of them require high processing capabilities, high computational costs and very low response times, which in many cases prevents its implementation
\end{abstract}

Key words: Learning, Resource Allocation, Quality of Service, Cognitive Radio, Software Defined Radio DSP, FPGA, Cognitive Satellite Networks, Base Station. 


\section{INTRODUCCIÓN}

En nuestros días, una de las características más importantes que los seres vivos poseen para garantizar su supervivencia, es la habilidad de adaptación a su entorno, es por ello que los sistemas, en especial los de telecomunicaciones no se encuentran exentos de esta situación.

El tema de reconfigurabilidad, adaptabilidad e inteligencia en la capa física implica dos tecnologías complementarias: Radio Definido por Software (SDR, Software Defined Radio) y Radio Cognitivo (CR, Cognitive Radio). El término "Software Radio" fue acuñado por Joseph Mitola III en 1.991 para referirse a un tipo de radio reprogramable o reconfigurable $(1,2)$. Posteriormente, en el año 1999, el mismo Mitola introdujo el término "Cognitive Radio" (3), considerándola como una tecnología de gran significado e importancia y que define a un radio que puede cambiar sus indicadores de operación teniendo en cuenta uno o varios parámetros que mida o cense en su entorno. Mitola hace además, una breve mención de cómo la radio cognitiva puede interactuar en el sistema a un nivel de alcance de una red cognitiva y termina reconociendo que los sistemas inalámbricos utilizan insuficientemente los recursos del espectro.

En la figura 1. Se muestra el diagrama en bloques de una estación terrena satelital de Radio Cognitivo propuesta, basada en la arquitectura en (4).

Los transceptores de CR deben diseñarse para que posean capacidades de cambiar sus parámetros de transmisión como resultado de las condiciones de propagación, bandas disponibles de frecuencia, recursos disponibles, calidad de servicio solicitada por el usuario, tipo de servicio, entre otras; de esta forma, el sistema de CR debe ser concebido como un sistema con capacidad de reconfiguración y autoconfiguración que puede de forma dinámica establecer sus parámetros de operación de acuerdo a los criterios implementados por los algoritmos de aprendizaje y adaptación buscando maximizar el desempeño del sistema y optimizar la utilización de los recursos disponibles de acuerdo a las condiciones del entorno de radio y a las necesidades del usuario. Estas características indican que el sistema de radio debe estar en capacidad de censar su entorno y tomar decisiones autónomas en correspondencia con la estrategia más apropiada de adaptación.

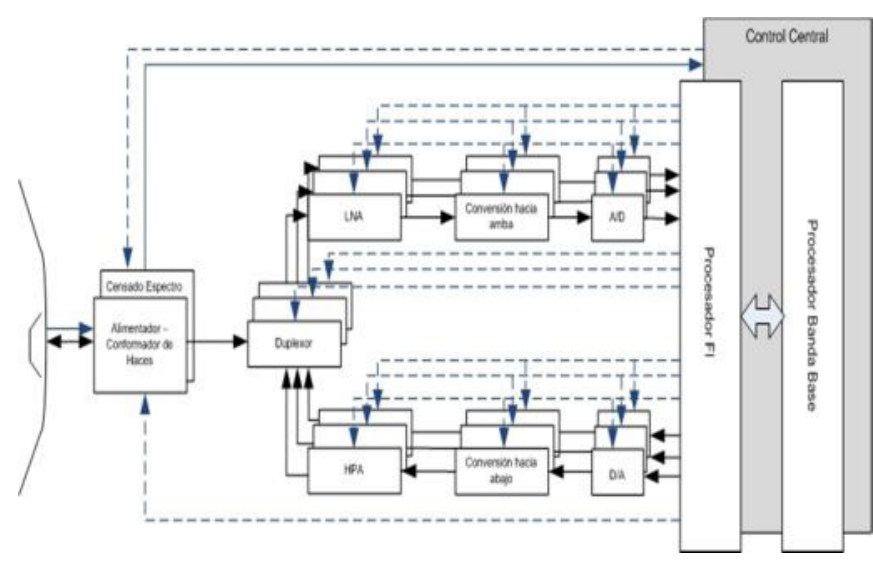

Figura 1. Estructura Básica de una Estación Terrena o Transpondedor Cognitivo.

Radio Cognitivo es una tecnología de radio condicionada al aprovechamiento del espectro no ocupado o que pueda ser compartido con el usuario que lo tiene legalmente asignado (usuario primario) sin perjudicar sus necesidades de comunicación. Esta forma de operación hace que radio cognitivo establezca sobre el nivel físico una nueva funcionalidad para la realización del censado de espectro sobre todos los grados de libertad disponibles (frecuencia, tiempo y espacio) para la identificación de los recursos espectrales disponibles o que pueden ser compartidos en la transmisión (2-4).

En este artículo a continuación se presenta algunos retos de diseño en las funciones de nivel físico al interior de un sistema satelital de radio cognitivo satelital incluyendo los dispositivos de radio del transceptor, circuitos de muestreo y de procesamiento digital y dispositivos de control. El eje fundamental de discusión es la funcionalidad del sistema satelital de radio cognitivo desde el enfoque de acceso al espectro y asignación dinámica de recursos de radio. De esta forma, en la sección 2 se aborda inicialmente la arquitectura propuesta y las funciones de nivel físico de una estación terrena satelital cognitiva, en la sección 3 se discute sobre las restricciones de diseño de nivel físico de la arquitectura y funciones de nivel físico en estaciones base y satélites cognitivos, en la sección 4 se presentan las funciones a implementar en las redes de radio cognitivo y en la sección 5 se presentan las conclusiones referentes a los retos de diseño identificados para este tipo de plataformas de radio global. 
Evaluación de restricciones de diseño de nivel físico de la arquitectura y funciones de nivel físico en redes satelitales cognitivas

En este aparte, se realiza una descripción de los retos que cada módulo en la cadena de RF y de control impone al diseño de una red satelital cognitiva y por consiguiente al diseño de la estación base y satélite cognitivo como el descrito en el apartado anterior (2).

La ampliación del ancho de banda de operación del sistema presenta las siguientes consecuencias:

- El número de portadoras de banda estrecha que ingresan a las cadenas de IF y banda base se incrementan de manera significativa, por lo tanto se incrementa el rango dinámico requerido en esta porción del sistema.

- La tasa de muestreo y el rango dinámico requerido para el conversor analógico a digital también debe incrementarse de manera significativa.

- La preselección de la portadora de RF se tornará compleja, ya que, los filtros de RF deben ser sintonizados a la banda de interés de acuerdo a la asignación de canal realizada por el controlador central.

- El duplexor debe tener frecuencias variables de operación y frecuencias variables de transmisión y recepción.

\section{Subsistema de Antenas.}

La arquitectura básica de SDR para un sistema de antenas inteligentes se muestra en la figura 2.Los flujos bidireccionales de datos indican la naturaleza recíproca de las funciones de procesamiento de datos. Esta arquitectura multiplica por $\mathrm{n}$ el número de transceptores $\mathrm{y}$ requiere que la capacidad de procesamiento de la plataforma hardware sea suficiente para la capacidad de tráfico del sistema, ya que permite expandir la capacidad del transpondedor con la cantidad de haces proporcionalmente conformables (2).

La arquitectura física debe permitir una suficiente flexibilidad de tal manera que pueda ser reconfigurado vía software y al mismo tiempo que sea fácilmente expandible para incrementar la capacidad del sistema.

El SDR Forum define un sistema de antenas inteligente como: "Un subsistema el cual incluye la antena que utiliza el dominio espacial en combinación con toma de decisiones basadas en procesamiento de señal para el mejoramiento de el desempeño del enlace y habilitar otros servicios de valor agregado." $(2,5)$. Lo anterior requiere elementos tanto hardware como software asociados con la capacidad de procesamiento adicional.

\section{Amplificador de Bajo Ruido}

El amplificador de bajo ruido (LNA, Low Noise Amplifier) permite elevar la potencia de la señal recibida a un rango compatible con otros módulos del sistema, minimizando los niveles de ruido presentes en la señal recibida. En este dispositivo, el reto primario consiste en maximizar su ganancia sin adicionar ruido excesivo a la señal, lo que puede ser conseguido sacrificando consumo de potencia y rango dinámico.

La idea es conseguir la mejora deseada del rango dinámico del LNA a través de redes de realimentación o control automático de ganancia con el fin de conseguir linealidad en el comportamiento del LNA sacrificando al mínimo la figura de ruido del sistema.

El AGC debe ser lo suficientemente rápido para detectar los cambios de nivel de la señal (posiblemente producidos por desvanecimiento) y realizar ajustes que no sean tan abruptos de tal manera que no introduzca distorsión. El AGC es necesario para garantizar que la señal no sea recortada por el ADC, pues, podría causar distorsión no lineal de la señal. Generalmente se incluye un AGC como parte del amplificador de bajo ruido en una cadena de RF en un transpondedor de tal manera que pueda ser controlado el nivel de ruido y distorsión introducidos por el amplificador al sistema, por lo cual permite mantener la figura de ruido constante mientras la ganancia en la cadena de recepción varía (2).

\section{Conversor Analógico a Digital - Conversor Digital Analógico}

El conversor analógico a digital (ADC) es el componente más difícil de seleccionar y el que impone las mayores restricciones de diseño en sistemas SDR, al punto que las restricciones del ADC probablemente se imponen en el diseño global de la arquitectura del sistema. Debe existir un compromiso entre las limitaciones reales del ADC tales como: tasas de muestreo, rango dinámico, resolución y consumo de potencia. El ADC debe muestrear una señal real a una tasa teórica que cumpla con la de Nyquist, y en el caso de sistemas multimodo, el gran ancho de banda a manejar determina la tasa de muestreo de las señales. Igualmente, señales de diferen- 
tes características o estándares requieren diferentes valores del rango dinámico, este típicamente se establece por la cantidad de interferencia de canal adyacente del sistema (2).

Una vez efectuada una detección confiable, un radio cognitivo debe seleccionar el esquema de transmisión adecuado para proporcionar la óptima utilización de recursos espectrales y de capacidad del canal asignado. En este punto, existen varios requisitos que el subsistema de modulación debe satisfacer. Primero, las bandas disponibles para transmitir pueden encontrarse dispersas en un amplio rango de frecuencias con anchos de banda variables; la distribución del espectro no ocupado o que puede ser compartido es una función de la ubicación geográfica y del tiempo de utilización de los usuarios primarios, esta distribución se debe actualizar en cada periodo de censado. Segundo, para garantizar una óptima utilización de recursos espectrales y de potencia, el radio cognitivo debe estimar la capacidad disponible de cada banda para proporcionar información de calidad de servicio a los niveles superiores. Por último, diferentes aplicaciones de usuario manejadas por la estación terrena cognitiva pueden requerir la asignación de bandas de frecuencia, lo cual implica diferentes requerimientos de calidad de servicio, por lo tanto el esquema de transmisión seleccionado debe permitir la asignación de cualquier banda de frecuencia a cualquier usuario y/o servicio y debe ser escalable con el número de usuarios y bandas disponibles (2).

\section{Controlador Central}

Las redes de radio cognitivo (CRNs - Cognitive Radio Networks) presentan retos únicos en la actualidad debido a la coexistencia con redes primarias y a la diversidad de requerimientos de calidad de servicio, por consiguiente, las funciones de gestión del espectro se tornan más complejas e introducen nuevos retos de diseño en los sistemas que conforman la red de radio cognitivo. Las redes de radio cognitivo deben evitar y/o mantener en niveles muy bajos la interferencia con las redes primarias, deben conocer la calidad de servicio requerida por los diferentes tipos de tráfico y mantener una comunicación fluida sin afectar las comunicaciones de los usuarios primarios.

A continuación se presenta las funciones a implementar en redes de radio cognitivo como los métodos para afrontar los anteriores retos, las funcionalidades requeridas a ser diseñadas para la gestión de recursos en los sistemas de radio cognitivo para su operación en una red de radio cognitivo son las siguientes:

- Censado de espectro: A un usuario de un radio cognitivo se puede asignar una porción de espectro no utilizada o disponible que satisfaga los requerimientos de calidad de servicio.

- Compartición de Espectro: Debido a que pueden existir múltiples usuarios cognitivos intentando acceder al espectro y/o capacidad disponible, en la red de radio cognitivo se debe coordinar el acceso para prevenir que múltiples usuarios cognitivos utilicen las mismas porciones de espectro y/o las mismas capacidades disponibles.

- Movilidad espectral: Los usuarios cognitivos son considerados como visitantes espectrales. Por consiguiente, si una porción específica del espectro que se encuentre en uso por parte de un usuario cognitivo es requerida por un usuario primario, la comunicación del usuario cognitivo debe continuar en otra porción del espectro a menos que el usuario cognitivo esté en capacidad de reconfigurar dinámicamente sus parámetros de transmisión de tal forma que la interferencia sobre el usuario primario sea tal que no afecte la comunicación de este último.

El desempeño de la red de radio cognitivo en aspectos relacionados con el rendimiento a nivel de tráfico se observan en la decisión de asignación, compartición y movilidad espectral. Todas estas funciones juntas son las responsables de garantizar un uso eficiente de los recursos de radio. Para conseguir esto, en la actualidad se está investigando en la solución de múltiples problemas relacionados con funciones de control a implementarse en las redes de radio cognitivo, entre ellas se tienen las siguientes.

\section{Selección de canal y Asignación de potencia basado en eficiencia energética}

En los sistemas de radio cognitivo existen dos modelos básicos de operación; Acceso Oportunístico de Espectro (OSA - Opportunistic Spectrum Access) y Compartición de Espectro (SS - Spectrum Sharing). En el modelo OSA los usuarios secundarios (usuarios cognitivos) pueden transmitir sobre las bandas de interés cuando los usuarios primarios no transmiten sobre dichas bandas; mientras que en el modelo SS se permite la transmisión de los usuarios secundarios simultáneamente con los usuarios primarios en la mismas bandas haciendo que los usuarios secundarios puedan controlar 


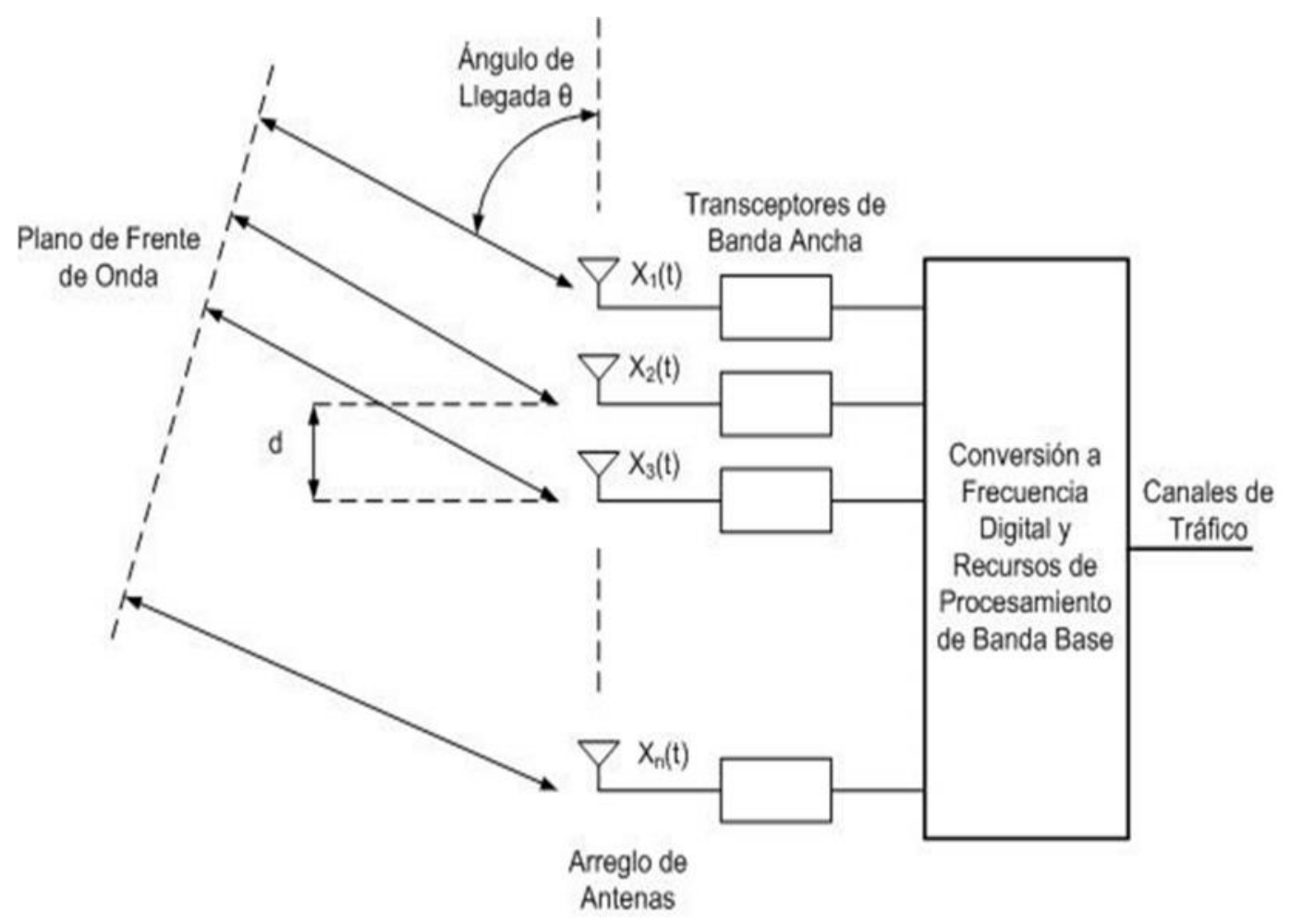

Figura 2. Arquitectura Básica de un Subsistema de Antenas Basado en SDR.



Figura 3. Modelo De Acceso Oportunístico Basado En Canal De Acceso Múltiple Vectorial. 
la interferencia que imponen a los usuarios primarios de tal forma que la degradación del desempeño de cada usuario primario se garantice está dentro de un margen tolerable. $(6-15)$.

Modelando la interacción entre un conjunto de $\mathrm{K}=\{1,2,3, \ldots, \mathrm{k}\}$ transmisores comunicándose con un único receptor a través de un conjunto de $\mathrm{N}=\{1,2,3, \ldots$ $, n\}$ canales ortogonales disponibles en el sistema como se muestra en la figura 3; se asume que los transmisores llegan secuencialmente a la red y el subíndice $\mathrm{k}$ indica el orden de llegada; de igual manera se asume que todos los transmisores se encuentran equipados con una única antena donde cada transmisor selecciona uno o varios canales en orden a minimizar la potencia requerida para garantizar una tasa de información constante dado el esquema de modulación codificación utilizado y sujeto a la limitación en potencia.

$$
\forall k \in K, \frac{1}{N} \sum_{n=1}^{N} p_{k, n} \leq p_{k, \max }
$$

Donde $p_{k, n}$ denota la potencia transmitida por el transmisor $\mathrm{k}$ sobre el canal n y $p_{\mathrm{k}, \max }$ denota el máximo nivel de potencia para transmisión disponible por el transmisor k.

En (16) se estudian las propiedades de la función de utilidad planteada, de donde se deriva que se observa un equilibrio de Nash cuando todos los transmisores alcanzan una SINR lo más cercano posible al valor óptimo denotado como $\gamma^{*}$, la cual se obtiene como solución de la ecuación.

$$
\gamma^{*} f^{\prime}\left(\gamma^{*}\right)-f\left(\gamma^{*}\right)=0
$$

Solución que corresponde con el único máximo de la función.

Si todos los jugadores intentan alcanzar la misma SINR $\gamma^{*}$ la potencia de transmisión óptima puesta por el jugador k sobre el canal $n$ está dada por

$$
p_{k, n}^{*}=\left(\frac{\sigma^{2}}{\left|h_{k, n}\right|^{2}}\right)\left(\frac{\gamma^{*}}{1-\alpha \theta(n) \gamma^{*}}\right)
$$


Considerando el escenario en el cual $\mathrm{N}=2$ y $\mathrm{K}=2$ se tiene

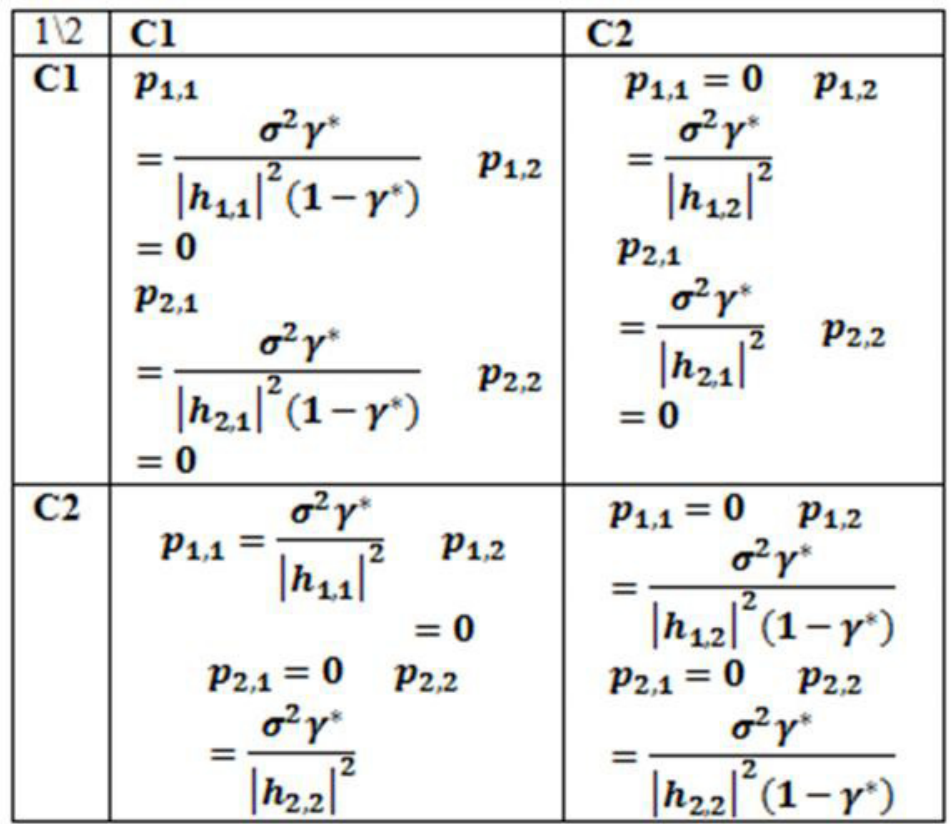

De donde se obtienen las siguientes regiones de equilibrio del juego $G=\left\{K, \boldsymbol{p}_{k}, u_{k}\left(\boldsymbol{p}_{k}, \boldsymbol{p}_{-k}\right)\right\}$ considerando que cada transmisor busca minimizar la potencia requerida para garantizar una tasa de información constante dado el esquema de modulación codificación.

Equilibrio $1(\mathrm{C} 1, \mathrm{C} 1)$

$$
\frac{\left|h_{1,2}\right|^{2}}{\left|h_{1,1}\right|^{2}}<1-\gamma^{*} \text { y } \frac{\left|h_{2,2}\right|^{2}}{\left|h_{2,1}\right|^{2}}<1-\gamma^{*}
$$

Equilibrio $2(\mathrm{C} 1, \mathrm{C} 2)$

$$
\frac{\left|h_{1,2}\right|^{2}}{\left|h_{1,1}\right|^{2}}<\frac{1}{1-\gamma^{*}} \text { y } \frac{\left|h_{2,2}\right|^{2}}{\left|h_{2,1}\right|^{2}}>1-\gamma^{*}
$$

Equilibrio $3(\mathrm{C} 2, \mathrm{C} 1)$

$$
\frac{\left|h_{1,2}\right|^{2}}{\left|h_{1,1}\right|^{2}}>1-\gamma^{*} \text { y } \frac{\left|h_{2,2}\right|^{2}}{\left|h_{2,1}\right|^{2}}<\frac{1}{1-\gamma^{*}}
$$

Equilibrio $4(\mathrm{C} 2, \mathrm{C} 2)$

$$
\frac{\left|h_{1,2}\right|^{2}}{\left|h_{1,1}\right|^{2}}>\frac{1}{1-\gamma^{*}} \text { y } \frac{\left|h_{2,2}\right|^{2}}{\left|h_{2,1}\right|^{2}}>\frac{1}{1-\gamma^{*}}
$$

\section{Calidad de Servicio}

El problema de proporcionar calidad de servicio en redes de radio cognitivo o auto configurables puede modelarse como un juego estático no cooperativo (6 -13) considerando $K$ pares de transmisores - receptores transmitiendo simultáneamente información independiente y sujetos a interferencia mutua. La métrica de calidad de servicio será su tasa de transmisión (función de utilidad) y el conjunto de acciones será el conjunto de diferentes niveles de potencia a transmitir. Cada transmisor debe garantizar una mínima tasa de transmisión denotada por $\Gamma_{\mathrm{k}}$ para el jugador $k$, si $h_{\mathrm{j}, \mathrm{k}}$ representa la realización del canal desde el transmisor $j$ hasta el receptor $k$ y asumiendo que los canales son invariantes en el tiempo durante el periodo de transmisión y denotando los símbolos transmitidos por el transmisor $k$ en el tiempo $t$ como $x_{k}(t)$, donde $p_{k}(t)=E\left[\left(x_{k}(t) x_{k}(t)^{*}\right)\right] \leq p_{k, \max }$ y la señal recibida por el receptor k se representa como $y_{k}(t)=\mathrm{h}_{k, k} x_{k}(t)+\sum_{j \neq k}{ }^{K} h_{k, j} x_{j}(t)+w_{\mathrm{k}}$ donde $w_{k}$ es una variable aleatoria con varianza $\sigma_{k}^{2}$ que representa la potencia de ruido en el receptor $k$, luego la función de utilidad del transmisor $k$ se define como. 
Donde la solución del problema será aquella en la cual los jugadores maximicen su utilidad satisfaciendo los requerimientos de calidad de servicio.

\section{Selección de Estación Base}

El escenario de selección de estación base puede modelarse de la misma manera como un juego no cooperativo en el cual el conjunto de transmisores $\mathrm{K}$ es el conjunto de jugadores, y el conjunto $\mathrm{S}$ es el conjunto de las estaciones base disponibles en la red (16-22) donde la estrategia de un jugador determinado es la potencia asignada mediante waterfilling denotada por el vector $\boldsymbol{p}_{k}=\left(p_{k, 1}, p_{k, 2}, \ldots, p_{k, S}\right)$ donde $\boldsymbol{p}_{\boldsymbol{k}}$ es el conjunto de todas las acciones del jugador $k$, considerando que cada jugador transmite únicamente a una estación base, la estrategia se define por un conjunto finito $\boldsymbol{p}_{\boldsymbol{k}}$.

La función de utilidad del jugador $k$ mide la satisfacción del jugador k con respecto a la estrategia seleccionada; definiendo esta función para todos los jugadores como su eficiencia espectral de la forma

$$
u_{k}\left(\boldsymbol{p}_{\boldsymbol{k}}, \boldsymbol{p}_{-\boldsymbol{k}}\right)=\sum_{S} \frac{B_{s}}{B} \log _{2}\left[\left(1+\gamma_{k, s}\right)\right]
$$

Donde $B_{s}$ es el ancho de banda disponible por la estación base $s$ y $B$ es el ancho de banda total disponible en la red $B=\sum_{\mathrm{S}} B_{s}$; la solución del problema será aquella en la cual los jugadores maximicen su utilidad (corresponderá a la estación base que garantiza mayor eficiencia espectral), la cual es de la forma

$$
p_{k, n}^{*}=\left[\frac{B_{s}}{B \beta_{k}}-\frac{\varepsilon_{k, S}}{g_{k, s}}\right]^{+}
$$

El cual es el único equilibrio de nash del juego. La constante $\beta_{k}$ para cada jugador $\mathrm{k}$ es el conjunto que satisface la condición

$$
\forall k \in K, \sum_{s=1}^{s} p_{k, s}=p_{k, \max }
$$

$\mathrm{Y} \varepsilon_{k, s}$ representa el ruido mas la interferencia en el canal de acceso múltiple superado por el jugador $k$ en la estación base $s$.

\section{CONCLUSIONES}

En este artículo se presentaron algunos retos en el diseño del nivel físico de redes satelitales cognitivas; primero se considera la implementación de las funciones de radio coherentes con radio cognitivo de tal forma que se puedan desempeñar funciones tales como la detección de usuarios primarios a través del censado de espectro; esta función de censado impone fuertes retos al diseño ya que requiere que los dispositivos de la cadena de radio trabajen adecuadamente en un amplio rango de frecuencias, (comercialmente hoy por hoy en la banda comprendida desde los $1.5 \mathrm{GHz}$ hasta los $30 \mathrm{GHz}$ ), además de la capacidad requerida de procesamiento de señal en los dispositivos de frecuencia intermedia y banda base.

Uno de los retos más importantes es la implementación de conversores analógico a digital y digital a analógico que permitan muestrear a una gran resolución una señal de banda ancha, lo cual implica, la necesidad de un gran rango dinámico de operación por parte de estos dispositivos, por lo cual se depende en gran medida de la evolución tecnológica en este tipo de dispositivos.

Otro reto importante está relacionado con la capacidad y velocidad de procesamiento del controlador central que se encarga de la totalidad de la coordinación y ejecución de las tareas de radio cognitivo en la estación base que propende por la óptima utilización de los recursos en la red cognitiva, como fue descrito en la sección 3 los algoritmos a implementar sobre el controlador central son complejos y deben permitir a la estación base satelital cognitiva tomar decisiones óptimas referentes a: la asignación de recursos, suministro de calidad de servicio, selección de la estación base (satélite), capacidad de aprendizaje a partir de la experiencia, etc.; funciones que deben realizarse en tiempo real.

Finalmente, se considera que CR es una tecnología promisoria la cual contribuirá al mejoramiento de los sistemas satelitales, sin embargo, su implementación puede tomar algún tiempo bastante largo dados los retos de diseño mencionados en las secciones anteriores. 


\section{BIBLIOGRAFÍA}

1. H. Harada and R. Prasad, Simulation and Software Radio for Mobile Communication, New York: Artech House Publishers, 2002; p. 467.

2. E. Astaiza H. and P. Vera, "Estudio de factibilidad de la aplicación de SDR a plataformas satelitales reconfigurables," Tesis de maestría - Universidad de Cauca, Popayán, 2008.

3. J. Mitola, "Cognitive radio for flexible mobile multimedia communications," Proc. IEEE Int. Workshop Mobile Multimedia Communications, 1999; pp. 3-10.

4. S. Medina, E. A. H. and P. V. V., "Reconfigurable satellite payload model based on software radio tecnologies," Quito, 2006.

5. S. Haykin, "Cognitive radio: Brain-Empowered wireless communications", 2005; 23(2).

6. H. Tang, "Some physical layer issues of wide-band cognitive radio system," in Fist IEEE International Symposium on New Frontiers in Dynamic Spectrum Access Networks, 2005.

7. L. Le and E. Hossain, "Resource allocation for spectrum underlay in cognitive radio networks," IEEE Transactions on Wireless Communications, 2008; 7(12), Part 2.

8. J. George, A. Sultan and M. Nafie, "Distributed admission and power control for cognitive radios in spectrum underlay networks," GLOBECOM proceedings, 2008.

9. D. I. Kim, L. Le and E. Hossain, "Joint rate and power allocation for cognitive radios in dynamic spectrum access environment," IEEE Transactions on Wireless Communications, 2008; 7(12), part 2.

10. S. Lasaulce, M. Debbah and E. Altman, "Methodologies for analyzing equilibria in wireless games," IEEE Signal Processing Magazine, Special issue on Game Theory for Signal Processing, 2009.

11. V. Srivastava, J. Neel, A. Mackenzie, R. Menon, L. Dasilva, J. Hicks, J. Reed and R. Gilles, "Using game theory to analyze wireless ad hoc networks," in IEEE Communications Surveys \& Tutorials, 2005.

12. S. Ross and B. Chaib-draa, "Satisfaction equilibrium : Achieving cooperation in incomplete information games," in 19th Canadian Conf. on Artificial Intelligence, 2006.

13. J.-S. Pang, G. Scutari, F. Facchinei and C. Wang, "Distributed power allocation with rate constraints in Gaussian parallel interference channels," IEEE Trans. on Info. Theory, 2008; 54(8), pp. 3471-3489.

14. P. Maille and B. Tuffin, "Optimization of transmission power in competitive wireless networks," Lecture notes in computer science, 2009.

15. W. Yu, W. Rhee, S. Boyd and J. Cioffi, "Iterative water-filling for Gaussian vector multiple-access channels," IEEE Transactions on Information Theory, 2004; pp. 145-152.

16. V. Rodriguez, "An Analytical Foundation for Resource Management in Wireless Communications," in IEEE Global Telecommunications Conference, San Francisco, 2003.

17. H. Lee, S. Kim, O. Lee, S. Choi and S.-J. Lee, "Available Bandwidth-Based Association in IEEE 802.11 Wireless LANs," in MSWiM '08 Proceedings of the 11th international symposium on Modeling, analysis and simulation of wireless and mobile systems, ACM New York, 2008.

18. L.-H. Yen, J.-J. Li and C.-M. Lin, "Stability and Fairness of Native AP Selection Games in IEEE 802.11 Access Networks," in Seventh International Conference On Wireless And Optical Communications Networks (WOCN) 20, 2010.

19. T. Bonald, A. Ibrahim and J. Roberts, "The Impact of Association on the Capacity of WLANs," in 7th International Symposium on Modeling and Optimization in Mobile, Ad Hoc, and Wireless Networks, WiOPT 2009.

20. V. Ghini, S. Ferretti and F. Panzieri, "A strategy for best access point selection," in Wireless Days (WD), 2010 IFIP.

21. I.-P. Hsieh, F.-M. Chang and S.-J. Kao, "Adaptive Access Points Selection for 802.11 Wireless Networks," in Proceeding AsiaCSN '07 Proceedings of the Fourth IASTED Asian Conference on Communication Systems and Networks, 2007.

22. V. S., P. K., D. C., K. J. and T. D., "Facilitating Access Point Selection in IEEE 802.11 Wireless Networks," in IMC "05 Proceedings of the 5th ACM SIGCOMM conference on Internet Measurement, 2005 . 